Saudi Journal of Medicine

Abbreviated Key Title: Saudi J Med ISSN 2518-3389 (Print) |ISSN 2518-3397 (Online) Scholars Middle East Publishers, Dubai, United Arab Emirates Journal homepage: https://saudijournals.com/sjm

Original Research Article

\title{
Clinical and Electrophysiological Correlation of Peripheral Neuropathy in Patients of Type 2 Diabetes Mellitus
}

\author{
Md. Eajaz Ahmed Shariff ${ }^{1 *}$, Fahad Al- Qahtani ${ }^{2}$ \\ ${ }^{1}$ Faculty of Dentistry, Division of Physiology, Department of Basic Medical Sciences, Al-Bahah University-65527, Kingdom of Saudi Arabia \\ ${ }^{2}$ Dean, Faculty of Dentistry, Al-Bahah University-65527, Kingdom of Saudi Arabia
}

DOI: $10.36348 / \mathrm{sjm} .2020 . \mathrm{v} 05 \mathrm{i} 04.008$

| Received: 23.03.2020 | Accepted: 02.04.2020 | Published: 21.04.2020

*Corresponding author: Md. Eajaz Ahmed Shariff

\section{Abstract}

We evaluated the prevalence of peripheral neuropathy in type 2 diabetes mellitus (T2DM) by clinical examination and nerve conduction study (NCS), and to correlate them with risk factors. 58 T2DM patients attending OPD in the department neurology of Jubilee mission medical college hospital, Thrissur were evaluated. Grading of signs and symptoms were assessed by Neuropathy symptoms score (NSS) and Neuropathy disability score (NDS) followed by nerve conduction studies. Diabetic status was evaluated by HbA1c levels and patients were categorized Group I HbA1c $<5$ (No diabetes), Group II- HbA1c= 5-7(Long standing controlled diabetes), Group III HbA1c $>7$ (Uncontrolled diabetes). $58 \mathrm{~T} 2 \mathrm{DM}$ patients of which $42(72 \%)$ were males and $16(28 \%)$ were females and their mean age were $52.1 \pm 8.8$ and 48.6 \pm 5.3 respectively. Clinical findings showed abnormal deep tendon reflexes in male 37(88\%) and female patients $11(66 \%)$. Deep sensory loss was highest among male 39(92\%) and female patients 12(75\%) respectively. Loss of pain and touch sensation male and female patients were found to be $24(57 \%), 9(56 \%)$ and $35(92 \%), 12(75 \%)$ respectively. Atrophy of the muscles in male and female patients were found to be $13(30 \%)$ and $4(25 \%)$ respectively. Electrophysiological assessment showed 34(58.6\%) patients in the severe group and score was 8 . However mild and moderate patients were $3(5.1 \%)$ and $21(36.2 \%)$ and there severity score was 5and 6 respectively.T2DM patients has high prevalence of diabetic neuropathy. Motor nerve conduction abnormalities were severe in males with diabetic peripheral neuropathy. Motor mononeuropathy reflects more severe involvement and peroneal and ulnar neuropathy is well marked in these patients.

Keywords: Diabetic peripheral neuropathy, Type 2 diabetes mellitus (T2DM), Glycated hemoglobin, Nerve conduction studies, Neuropathy symptoms score (NDS), Neuropathy Disability score (NDS).

Copyright @ 2020: This is an open-access article distributed under the terms of the Creative Commons Attribution license which permits unrestricted use, distribution, and reproduction in any medium for non-commercial use (NonCommercial, or CC-BY-NC) provided the original author and source are credited.

\section{INTRODUCTION}

Diabetes mellitus is a chronic illness characterized by increased risk of macrovascular and microvascular complications and linked with longstanding injury and malfunction of different organs like the eyes, kidneys, skin, heart, nerves, and blood vessels $[1,2]$.

T2DM is a heterogeneous group of disorders characterized by combination of Insulin resistance and an Insulin secretory defect [3-7]. The Majority of patients about $90-95 \%$ have type 2 DM. T2DM treated with diet modification and regular exercise, moreover, diet is supplemented with oral hypoglycemic agents or may require parenteral insulin for persisting hyperglycemia. In a diabetic world, Peripheral neuropathies foremost and frequent complications.
Diabetic peripheral neuropathy is defined as "the presence of symptoms and or signs of peripheral nerve dysfunction in people with diabetes after exclusion of other causes" [8]. Neuropathies are the most common complication affecting up to $50 \%$ of patients with type 1 and type 2 DM which decreases their quality of life [9-11]. All of the axons are affected either by Wallerian degeneration. This is true for diabetic patients, and other metabolic neuropathies, in which conduction velocities range from normal to mildly slow. In these axonal neuropathies, the motor and sensory nerve amplitudes are diminished [20].

Diabetic neuropathy manifest with a wide variety of sensory, motor, and autonomic symptoms and classified into several clinical syndromes (American Diabetes Association, (APA) [12]. The primary symptoms of neuropathy include burning, stabbing down the leg, pins, needles and tingling sensation) are highly unpleasant, while the secondary complications 
are even more severe and can lead to fractures, amputations, and even death in patients with DM [13, 14]. The evaluation of DPN is based on patient symptoms and physical examination, simple screening methods [15]. Early diagnosis and management are crucial to failure to diagnose diabetic polyneuropathy can lead to serious consequences, including disability and amputation [13].

Nerve Conduction Studies measures the ability of the nervous system to conduct electrical impulses and to evaluate nerve/muscle function to determine the presence of neuromuscular disease. It is highly sensitive for diagnosing DPN. Nerve conduction studies (NCS) and its correlates in patients with DPN is crucial for early diagnosis and development of active management strategies and prevention of its dreaded complications [16].

Diabetic polyneuropathy is an often an undetected condition and there is a need for the early diagnosis and management in real practice, our current study is an attempt toward it to fulfill this objective, to study prevalence of peripheral neuropathy in type 2 diabetes mellitus (T2DM) by clinical examination and nerve conduction study (NCS), and to correlate them with risk factors.

\section{MATERIAL AND METHODS}

This was a cross-sectional study conducted in the department of physiology and neurology of Jubilee mission Medical college hospital, Thrissur. Institutional ethical committee clearance was obtained prior to the commencement of the study.

\section{Study Design}

58 T2DM patients with symptoms of diabetic peripheral neuropathy attending out patient's neurology clinics were recruited for the study. Written informed consent was obtained prior to the beginning of the study.

\section{Clinical Evaluation}

Detailed medical history was taken, and complete clinical examination was done in all patients. Baseline measurements of blood pressure, height, weight and body mass index (BMI) were done.

\section{Inclusion Criteria}

Patients with T2DM with symptoms of tingling sensation in the foot, loss of sensation, burning feet, paresthesia, muscle weakness, muscle wasting, muscle atrophy, foot ulcers etc. Age group 18-60 years of age of either gender were included.

\section{Exclusion Criteria}

Patients with Type1 DM, Cerebrovascular Accidents (CVA), limb Ischemia, Chronic Alcoholic etc.
Symptoms among the patients were evaluated using the Neuropathy Symptom Score (NSS). All patients were asked whether they experienced paresthesia in their legs. A description of burning, numbness, or tingling was assigned a score of 2 , and fatigue, cramping, or aching was assigned a score of 1 . If the patient described the symptoms as occurring in their feet, calves, and elsewhere, scores of 2,1 , and 0 were assigned, respectively. Nocturnal exacerbation of symptoms was scored as 2; exacerbation of symptoms during the day as well as night was scored as 1 , and exacerbation of symptoms during the daytime alone was scored as 0 . If the symptoms had ever awakened the patient from sleep, a score of 1 was assigned.

The patients were asked if any manoeuvre could reduce their symptoms; walking was assigned a score of 2 , standing 1 , and sitting or lying down 0 . Thus, the maximum symptom score was 9 . A minimum score of 3 was taken as the cut off to label a patient to have clinical neuropathy. Severity of symptoms were graded as: mild (scores: 3-4), moderate (scores: 5-6), and severe (scores: 7-9).8 similarly, the signs were graded using the Neuropathy Disability Score [25]. Examination of vibration (using a $128-\mathrm{Hz}$ tuning fork), pin-prick sensation (using pin), temperature sensation (using warm and cool water filled test tubes), and Achilles tendon reflex (using a tendon hammer) were done. The 3 perceptions were scored 0 if present and normal, and 1 if absent, reduced, or uncertain. On either side, the ankle reflex was scored 0 if present and normal, and 2 if absent. The maximum score was 10 . A minimum score of 3 was taken as the cut off to label a patient to have clinical neuropathy. The severity of NDS graded as, mild (scores: 3-5), moderate (scores: 6-8), and severe (scores: 9-10).

Diabetic status was evaluated by HbA1c levels and patients were categorized into 3 groups. Group I HbA1c $<5$ (No diabetes), Group II- HbA1c= 5-7(Long standing controlled diabetes), Group III HbA1c >7 (Uncontrolled diabetes).

\section{HbA1c}

The HbA1c levels were determined by the borate affinity assay (Nyco Card, AXIS-SHIELD PoC AS, Norway). Briefly $2 \mathrm{ml}$ of blood was collected in EDTA tube, HbA1c were assayed as per instructions supplied with the kit [2].

\section{Electrophysiological Assessment Nerve Conduction Studies}

Nerve conduction study measurements were performed in a warm room with the participants in a supine position. Nerve conduction velocity data were recorded using an RMS EMG EP Mark II instrument (Recorders and Medicare System, Chennai, India). First of all a written consent was taken from each subject. The skin temperature was maintained within 36 - 38 degrees centigrade. No skin preparation was 
needed. Filters were set at 2 Hertz to 5 Hertz and sweep speed was 5 millisecond per division for motor study and for sensory study, filters were set at 20 Hertz to 3 Kilo Hertz and sweep speed was 2 millisecond per division. Motor conduction study was performed on the median nerve in the upper limb and on common peroneal nerve in the lower limb. Sensory conduction study was performed on the ulnar nerve in the upper limb and on sural nerve in the lower limb. The targeted nerve was supra maximally stimulated using a square wave current with a duration of 0.2 millisecond. The length of each nerve was measured with a flexible tape. For median motor nerve conduction study, the recording electrode was placed close to the motor point of abductor pollicis brevis and reference electrode 3 centimeters distal at first metacarpophalangeal joint. The ground electrode is placed between the recording electrode and the stimulating electrode. A supra maximal stimulation is given at 3 centimeters proximal to the distal wrist crease and at the elbow. The distal latency, nerve conduction velocity of different segments and compound muscle action potentials are measured. For the ulnar sensory nerve conduction the active ring electrode was placed over the fifth digit to record the responses, the reference electrode was placed 4 centimeters distal to the active electrode and the stimulation was performed 10 centimeters proximal to the active electrode and medial to the flexor carpi ulnaris tendon. Latency and sensory nerve action potential (SNAP) amplitude were measured and nerve conduction velocity calculated. For the motor peroneal nerve conduction study surface recordings are obtained from extensor digitorum brevis and stimulation is given at ankle, 2 centimeters distal to the fibular neck, at the fibula and 5 centimeters above the fibular neck. Latency and amplitude of compound muscle action potentials (CMAP) are measured and nerve conduction velocity calculated. Sural nerve is purely a sensory nerve. The sural nerve conduction velocity was measured antidromically. The active recording electrode was placed just under the lateral malleolus. The stimulating electrode was placed at a distance of 14 centimeters from the recording electrode. Latency and sensory nerve action potential amplitude were measured and nerve conduction velocity calculated [2, 20]. Electrophysiological assessment done by severity of nerve conduction velocity into mild, moderate and severe and were assessed by Neuropathy disability score [25].

\section{Statistical Analysis}

The data was tabulated by excel and summarized to test the difference in the mean values between the groups by using the one-way Anova. Graph Pad Prism 5 was used to compute the results and plot the graph.

\section{RESULTS}

58 diabetic peripheral neuropathy (DPN) patients participated in this study of which $42(72 \%)$ were males and 16(28\%) were females (Fig-1). The mean age group of the male and female patients were $52.1 \pm 8.8$ and $48.6 \pm 5.3$ respectively (Fig-2, Table-1). The average duration of diabetes of the male patients were $15.2 \pm 6.4$ years and the female patients were 13.6 \pm 2.5 years [Table 1]. In this study DPN cases were segregated into 3 groups based on HbA1c levels. Group $\mathrm{I}-\mathrm{HbA} 1 \mathrm{c}<5$ (No diabetes), Group II- HbA1c= 5-7 (Long standing controlled diabetes), Group III HbA1c $>7$ (Uncontrolled diabetes). Most of the patients $47(81 \%)$ enrolled in the study with uncontrolled diabetes and peripheral neuropathy were found to be in group III (HbA1c $>7)$.Patients with Group II (HbA1c=5$7)$ and Group I $(\mathrm{HbA} 1 \mathrm{c}<5)$ were found to be $13.7 \%$ and $5 \%$ respectively (Table- 2 ).

Study of motor and sensory signs in T2DM with peripheral neuropathy in male and female patients (Fig-3) showed abnormal deep tendon reflexes in male patients $37(88 \%)$ in comparison with the female patients $11(66 \%)$.Deep sensory loss was highest among male patients $39(92 \%)$ in comparison with the female counterparts $12(75 \%)$.Loss of pain and touch sensation male and female patients were found to be 24(57\%), $9(56 \%)$ and $35(92 \%), 12(75 \%)$ respectively. Atrophy of the muscles in male and female patients were found to be $13(30 \%)$ and $4(25 \%)$ respectively.

Electrophysiological grading was classified on the basis severity of nerve conduction velocity into mild, moderate and severe and were assessed by Neuropathy symptoms score and neuropathy disability score [25]. 34 patients were in severe group accounting $58.6 \%$ and severity score was 8 . However mild and moderate patients were $3(5.1 \%)$ and $21(36.2 \%)$ and there severity score was 5 and 6 respectively (Table-3).

Motor nerve conduction abnormalities were more frequent and severe in males with diabetic peripheral neuropathy. Peroneal nerve showed decreased excitability with reduced compound muscle action potential (CMAP, $(54.7 \%)$, absent or reduced CMAP of Tibial nerve found to be $40.4 \%$ and $19.0 \%$ respectively (Table-4). Ulnar axon loss and severely decreased ulnar nerve conduction velocities were almost two times more frequent and reduced median conduction velocity was more frequent in males. Absence of peroneal nerve motor response was a part of length dependent polyneuropathy (PNP) rather than focal entrapment mononeuropathy. However, prolonged distal latency of median palmar nerve was predominant in females (reduced CMAP of 31.2\%) and showed focal involvement at the wrist. Peroneal nerves showed decrease in nerve conduction velocity in comparison to the tibial and sural nerve (Table-5). 
Fig:1 Gender distribution of Diabetic patients

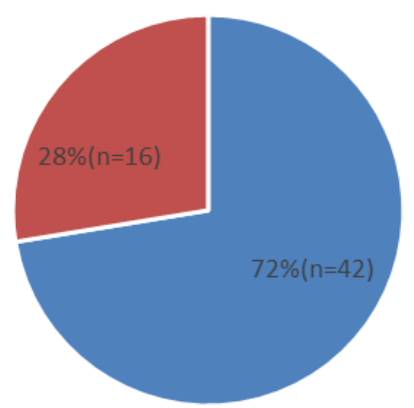

- Male

- Female

Fig-1: Gender distribution of Diabetic Patients

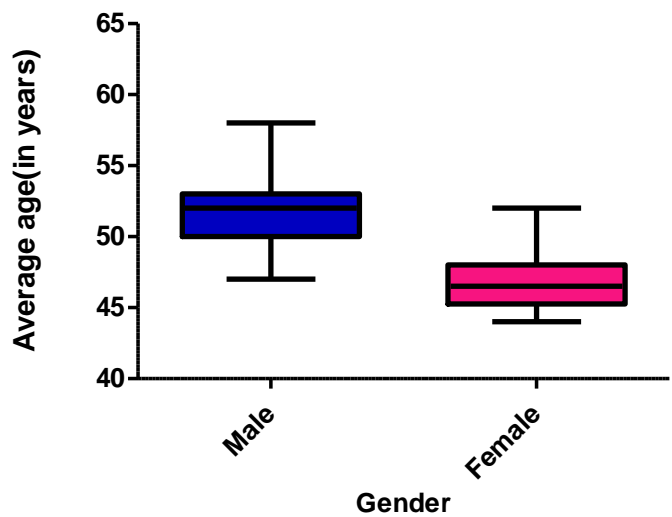

Fig 2: Average age of Type2 DM patients with peripheral neuropathy

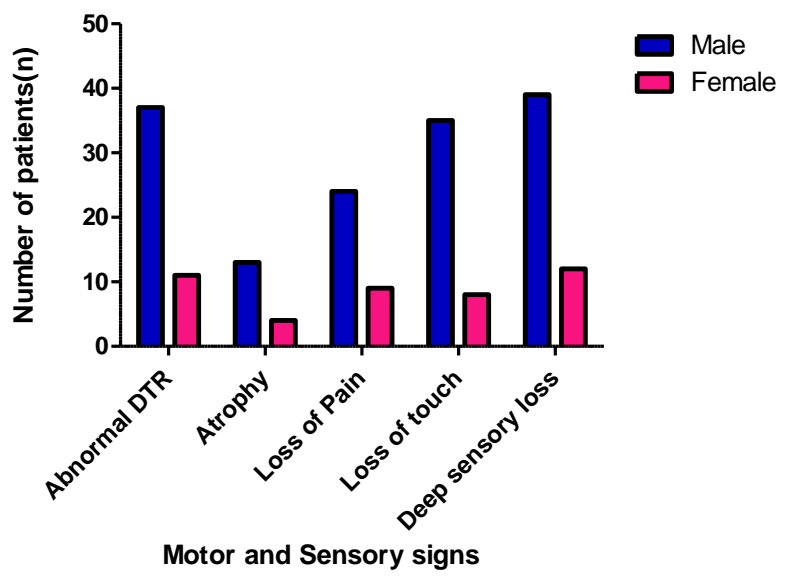

Fig 3: Motor and sensory signs in Type 2 DM patients with Peripheral neuropathy

Table-1: Average age and duration of diabetes in Type2 DM patients with diabetic neuropathy

\begin{tabular}{|l|l|l|l|}
\hline & Male & Female & Total \\
\hline Number of Patients (n) & 42 & 16 & 58 \\
\hline Mean age of the patients & $52.1 \pm 8.8$ & $48.6 \pm 5.3$ & $50.4 \pm 6.3$ \\
\hline Average duration of Diabetes & $15.2 \pm 6.4$ & $13.6 \pm 2.5$ & $14.4 \pm 2.4$ \\
\hline
\end{tabular}


Table-2: Diabetic profile in Type2 DM patients with Peripheral neuropathy

\begin{tabular}{|l|l|l|}
\hline Diabetic Profile(HbA1c) & \multicolumn{2}{|l|}{ Patients } \\
\hline Groups & $\mathrm{n}=58$ & $\%$ \\
\hline HbA1c $<5$ & 3 & 5.1 \\
\hline HbA1c $=5-7$ & 8 & 13.7 \\
\hline HbA1c $>7$ & 47 & 81 \\
\hline
\end{tabular}

Table-3: Relation of Clinical severity and Electro diagnostic nerve muscle grading

\begin{tabular}{|l|l|l|l|l|}
\hline $\begin{array}{l}\text { Classification } \\
\text { of severity }\end{array}$ & Electrophysiological grading & $\mathbf{n = 5 8}$ & $\mathbf{\%}$ & $\begin{array}{l}\text { Neuropathy disability } \\
\text { score (NDS) }\end{array}$ \\
\hline Mild & Slowing of sensory nerve, normal motor conduction & 3 & 5.1 & 5 \\
\hline Moderate & Prolongation of motor and sensory conduction & 21 & 36.2 & 6 \\
\hline Severe & Sensory nerve Action potential/CMAP* absent & 34 & 58.6 & 8 \\
\hline
\end{tabular}

*Compound Muscle action potential

Table-4: Electrophysiological findings in Male and Female in Type 2 DM patients with peripheral neuropathy

\begin{tabular}{|l|l|l|l|l|}
\hline Electrophysiological findings & \multicolumn{2}{|l|}{ Male } & \multicolumn{2}{l|}{ Female } \\
\hline Nerves & $\mathrm{n}=42$ & Percentage (\%) & $\mathrm{n}=16$ & Percentage (\%) \\
\hline Peroneal Nerve & & & & \\
\hline Absent & 19 & 45.2 & 2 & 12.5 \\
\hline Reduced CMAP** amp & 23 & 54.7 & 7 & 43.7 \\
\hline MCV< 70\% of Normal range & 27 & 64.2 & 5 & 31.2 \\
\hline Tibial Nerve & & & & \\
\hline Absent & 17 & 40.4 & 4 & 25 \\
\hline Reduced CMAP amp & 8 & 19.0 & 2 & 12.5 \\
\hline MCV<70\% of Normal range & 7 & 16.6 & 3 & 18.7 \\
\hline Median Nerve & & & & \\
\hline Absent & - & - & - & - \\
\hline Reduced CMAP amp & 4 & 9.5 & 5 & 31.2 \\
\hline MCV*<70\% of Normal range & 28 & 66.6 & 7 & 43.75 \\
\hline Ulnar Nerve & & & & \\
\hline Absent & - & - & - & - \\
\hline Reduced CMAP amp & 14 & 33.3 & 6 & 37.5 \\
\hline MCV< 70\% of Normal range & 31 & 73.8 & 8 & 50 \\
\hline
\end{tabular}

**compound muscle action potential, *Motor conduction velocity

Table-5: Comparison of electrophysiological findings in different Nerves of lower limb

\begin{tabular}{|l|l|l|l|}
\hline Nerve & Amplitude (\%) & Latency (\%) & NCV (\%) \\
\hline Right Tibial & 42.2 & 4.2 & 27.4 \\
\hline Left Tibial & 49.8 & 5.8 & 29.6 \\
\hline Right Peroneal & 69.0 & 36.2 & 41.0 \\
\hline Left Peroneal & 74.2 & 30.6 & 53.0 \\
\hline Right Sural & 48.2 & 18.6 & 26.6 \\
\hline Left sural & 50.2 & 22.6 & 25.0 \\
\hline
\end{tabular}

\section{DISCUSSION}

One of the feature of diabetic nerve damage is abnormality of sensory and motor nerve conduction An electrophysiological examination is a non-invasive approach in the assessment of peripheral nerve function. Population-based and clinical studies have been conducted to authenticate the sensitivity of these methods [17]. Our study showed there was higher prevalence of DPN in male patients and they were on treatment with oral hypoglycemic agents. Majority of our patients were males $(72 \%)$ and their mean age group found to be $52.1 \pm 8.8$ in comparison to the female $(28 \%)$ counterparts and their mean age group found to be $48.6 \pm 5.3$ respectively. Our findings were in consistent with other studies [18].

T2DM cases were segregated into 3 groups based on HbA1c levels. Group I-HbA1c $<5$ (No diabetes), Group II- HbA1c= 5-7 (Long standing controlled diabetes), Group III HbAlc >7 (Uncontrolled diabetes). Most of the patients 47(81\%) enrolled in the study with uncontrolled diabetes and peripheral neuropathy were found to be in group III (HbAlc $>7)$. Patients with Group II $(\mathrm{HbA} 1 \mathrm{c}=5-7)$ and Group I $(\mathrm{HbA} 1 \mathrm{c}<5)$ were found to be $13.7 \%$ and $5 \%$ respectively. In our study the average duration of diabetes of the male patients were $15.2 \pm 6.4$ years and 
the female patients were $13.6 \pm 2.5$ years. Our findings were in concordance with other studies as reported by Patil et al., and Kumar et al., [3, 19].

In the present study, motor and sensory signs in T2DM with peripheral neuropathy in male and female patients showed abnormal deep tendon reflexes in male patient's. Deep sensory loss was highest among male patients in comparison with the female counterparts. Loss of pain and touch sensation, muscle atrophy was more pronounced in male patients .Our findings were in agreement with other studies as reported by Kumar et al., [19].

Nerve conduction studies showed 34 patients were in severe group accounting $58.6 \%$ and severity score was 8 . However mild and moderate patients were $3(5.1 \%)$ and $21(36.2 \%)$ and there severity score was 5 and 6. Motor nerve conduction abnormalities were more common and severe in males with diabetic peripheral neuropathy. Our findings were in agreement with other studies $[2,4]$.

Our findings were peroneal nerve showed decreased excitability with reduced CMAP (54.7\%), absent or reduced CMAP of Tibial nerve found to be $40.4 \%$ and $19.0 \%$ respectively. Ulnar axon loss and severely decreased ulnar nerve conduction velocities were almost two times more common and reduced median conduction velocity was more common in males. Absence of peroneal nerve motor response was a part of length dependent polyneuropathy (PNP) rather than focal entrapment mononeuropathy. However, prolonged distal latency of median palmar nerve was predominant in females (reduced CMAP of $31.2 \%$ ) and showed focal involvement at the wrist.

Peroneal nerves showed decrease in nerve conduction velocity in comparison to the tibial and sural nerve. Similar findings were reported by other studies [4, 21-23]. Which replicates that the tibial nerve, sural nerve and planter nerve are the best nerves chosen for assessment of NCV studies in diabetic neuropathy. Tingling sensation, burning feet, diminished light touch and diminished vibration sensation are significantly associated with NCV in different nerves. These findings were in line with other studies, it signifies the role of $\mathrm{NCV}$ in the patient of diabetes, which can be used to detect neuropathy early with high sensitivity and specificity $[16,23]$. If any diabetic patient presenting with symptoms suggestive of neuropathy, NCV can be used early detection and management of it which can help to prevent any complication related to it. Negrin and Zara [24] reported that only severe peroneal nerve involvement has a prognostic value when it is considered together with clinical neurological signs in the natural history of diabetic neuropathy.

\section{CONCLUSION}

DPN is highly dependent neuropathy, lower extremity nerves are probably more involved. Nerve conduction studies having high sensitivity and specificity. Diabetic neuropathy is proportion to duration of diabetes. Severity of diabetic neuropathy was positively relating with higher blood glucose level. Paresthesia's and burning feet were most common found symptoms. Abnormal tendon reflexes and deep sensory loss were most common found signs and distal symmetrical types of polyneuropathy was the common types in our study. Peroneal, tibial and sural nerve were more commonly involved. Hence, authors propose that early detection of diabetic neuropathy through detailed assessment of lineal features and nerve conduction velocity testing of diabetes patients, to reduce the burden of complication in the form of neuropathy and to improve the quality of life in diabetics.

\section{Conflict of Interest: None \\ Funding: None}

\section{ACKNOWLEDGEMENTS}

I extend my gratitude and thanks for the Doctors, Nursing staff of Jubilee Mission Hospital, Thrissur for all the technical support during the course of the study.

\section{REFERENCES}

1. Sen, S., Chakraborty, R., \& De, B. (2016). Diabetes mellitus in 21st century (p. 186). Springer.

2. Bin-Jaliah, I. M., Shariff, M. E. A., Nayar, P. S., \& Chandramoorthy, H. C. (2016). Elevated basal cytosolic calcium of endothelial cells influences the post-surgical outcome in diabetic CTS. Physiology international, 103(4), 469-480.

3. Kakrani, A. L., Gokhale, V. S., Vohra, K. V., \& Chaudhary, N. (2014). Clinical and nerve conduction study correlation in patients of diabetic neuropathy. J Assoc Physicians India, 62(1), 24-7.

4. American Diabetes Association. (2018). 2 Classification and Diagnosis of Diabetes: Standards of Medical Care in Diabetes-2018. Diabetes Care. 41(1):13-27.

5. DeFronzo, R. A., Ferrannini, E., Groop, L., Henry, R. R., Herman, W. H., Holst, J. J., \& Simonson, D. C. (2015). Type 2 diabetes mellitus. Nat Rev Dis Primers. 1:1509.

6. Inzucchi, S. E., Bergenstal, R. M., Buse, J. B., Diamant, M., Ferrannini, E., Nauck, M., ... \& Matthews, D. R. (2015). Management of hyperglycemia in type 2 diabetes, 2015: a patientcentered approach: update to a position statement of the American Diabetes Association and the European Association for the Study of Diabetes. Diabetes care, 38(1), 140-149.

7. Chen, L., Magliano, D. J., \& Zimmet, P. Z. (2012). The worldwide epidemiology of type 2 diabetes mellitus-present and future 
perspectives. Nature reviews endocrinology, 8(4), 228-236.

8. Boulton, A. J., \& Malik, R. A. (1998). Diabetic neuropathy. Medical Clinics of North America, 82(4), 909-929.

9. Bansal, D., Gudala, K., Muthyala, H., Esam, H. P., Nayakallu, R., \& Bhansali, A. (2014). Prevalence and risk factors of development of peripheral diabetic neuropathy in type 2 diabetes mellitus in a tertiary care setting. Journal of diabetes investigation, 5(6), 714-721.

10. Kim, S. S., Won, J. C., Kwon, H. S., Kim, C. H., Lee, J. H., Park, T. S., ... \& Cha, B. Y. (2014). Prevalence and clinical implications of painful diabetic peripheral neuropathy in type 2 diabetes: results from a nationwide hospital-based study of diabetic neuropathy in Korea. Diabetes research and clinical practice, 103(3), 522-529.

11. Kiani, J., Moghimbeigi, A., Azizkhani, H., \& Kosarifard, S. (2013). The prevalence and associated risk factors of peripheral diabetic neuropathy in Hamedan, Iran. Archives of Iranian medicine, 16(1), 17-19.

12. American Diabetes Association. (2018). 2. Classification and diagnosis of diabetes: standards of medical care in diabetes-2018. Diabetes care, 41(Supplement 1), S13-S27.

13. Albers, J. W., \& Pop-Busui, R. (2014). Diabetic neuropathy: mechanisms, emerging treatments, and subtypes. Current neurology and neuroscience reports, 14(8), 473.

14. Martin, C. L., Albers, J. W., Pop-Busui, R., \& DCCT/EDiC research Group. (2014). Neuropathy and related findings in the diabetes control and complications trial/epidemiology of diabetes interventions and complications study. Diabetes care, 37(1), 31-38.

15. Malik, R. A. (2014). Which test for diagnosing early human diabetic neuropathy?. Diabetes, 63(7), 2206-2208.

16. Kimura, J. (2017). Nerve conduction studies. Oxford Textbook of Clinical Neurophysio, 49.

17. Najafi, M., Hasanzadeh, G., Hadian, M. R., Musa, A. E., \& Shirazi, A. (2018). Electrophysiological measurements of diabetic peripheral neuropathy: A systematic review. Diabetes \& Metabolic
Syndrome: Clinical Research \& Reviews, 12(4), 591-600.

18. Gul, S., Dar, M. A., Ali, A., Ashraf, A., \& Qadri, S. (2017). Prevalence of peripheral neuropathy and pattern of involvement on nerve conduction study in patients of Type 2 diabetes mellitus with clinical symptoms of peripheral neuropathy in kashmiri population a hospital based study. Imp $J$ Interdiscip Res, 3(2).

19. MH, S. K., Moosabba, M. S., \& Koppad, S. N. (2018). A study on peripheral neuropathy in patients with diabetic foot ulcers. International Surgery Journal, 5(3), 913-916.

20. Paleti, N. K., \& Elizabeth, B. (2018). A comparative study of nerve conduction velocities in human beings of different age. International Journal of Contemporary Medical Research 2018;5(8):1-6.

21. Albers, J. W., \& Pop-Busui, R. (2014). Diabetic neuropathy: mechanisms, emerging treatments, and subtypes. Current neurology and neuroscience reports, 14(8), 473.

22. Martin, C. L., Albers, J. W., Pop-Busui, R., \& DCCT/EDiC research Group. (2014). Neuropathy and related findings in the diabetes control and complications trial/epidemiology of diabetes interventions and complications study. Diabetes care, 37(1), 31-38.

23. Squintani, G., Zoppini, G., Donato, F., Pineschi, E., Donini, D., Stoico, V., ... \& Morini, A. (2014). Antidromic sensory nerve conduction study of the digital branches of the medial plantar nerve: a novel method to detect early diabetic sensory axonal polyneuropathy. Muscle \& nerve, 50(2), 193-199.

24. Negrin, P., \& Zara, G. (1995). Conduction studies as prognostic parameters in the natural history of diabetic neuropathy: a long-term follow-up of 114 patients. Electromyography and clinical neurophysiology, 35(6), 341-350.

25. Bhuyan, A. K., \& Appaiah, S. (2018). Clinical and electrophysiological correlation of peripheral neuropathy in newly diagnosed type 2 diabetes mellitus. International Journal of Advances in Medicine. 5:542-49. 